\title{
Image-Based Predictive Maintenance Concept for Inkjet Printing of Ceramic Inks
}

\author{
Peter Bischoff', Christoph Zeh ${ }^{1}$, Christoph Kroh ${ }^{2}$, Christiane Schuster ${ }^{1}$, Thomas Härtling ${ }^{1,3}$ \\ ${ }^{1}$ Fraunhofer Institute for Ceramic Technologies and Systems IKTS, Maria-Reiche-Str. 2, \\ 01109 Dresden, Germany, \\ 2 Senodis Technologies GmbH, Manfred-von-Ardenne-Ring 20D, \\ 01109 Dresden, Germany, \\ 3 Technische Universität Dresden, Institute of Solid State Electronics, Mommsenstraße 15, \\ 01069 Dresden, Germany \\ peter.bischoff@ikts.fraunhofer.de
}

\begin{abstract}
Summary:
Ceramic inks can be used to mark metal sheets in hot forming for track-and-trace purposes. However, the ceramic pigments in the inks can lead to clogging of printer nozzles which results in loss of print quality. Here we report on a predictive maintenance concept including different machine- and deeplearning models as the basis of a print quality assurance strategy. Pixelwise image segmentation leads to detailed information about the printing results. The information is used to train a model, classifying the remaining useful lifetime until insufficient printing results.
\end{abstract}

Keywords: predictive maintenance, machine learning, inkjet printing, ceramic pigments, image analysis

\section{Introduction}

By printing individual part markings (e.g. data matrix codes) on metal sheets as a first step in production, continuous tracking and tracing of all produced components can be achieved. A ceramic ink enables part marking in production environments where conventional marking methods are not appliable, e.g. in hot forming. Ceramic particles are added to the ink to ensure code readability after heating the metal sheets to temperatures up to $1200^{\circ} \mathrm{C}$, which is necessary for the forming process.

Due to the solid particles in the ink, several problems occur in the printing process. During long-term printing particles accumulate around the edges of the printing nozzles, affecting the inkjet. This leads to degrading printing quality and ultimately to non-readable part markings. At a testing station set up at Fraunhofer IKTS long-term testing is performed by regularly printing a test pattern. Typical printing faults observed are missing droplets in the printed test grid, large deviations compared to the desired printing position of the droplets, and the ejection of too little ink volume resulting in small and hardly visible droplets after heating the metal sheets. Currently no concept for detecting these flaws, especially in their early stages, exists, hence no automatic maintenance to restore the initial printer conditions can be per- formed. To address this challenge we implemented machine-learning methods and algorithms to quantify the state of the printer and to train a model which is able to reliably predict the remaining-useful-lifetime (RUL) until the quality of the printing results are not sufficient, i.e. not readable, any more.

\section{Methods}

To receive reliable data from images taken of the printed test pattern at the test station an image-segmentation model is implemented. The model is based upon the U-Net architecture and reduced in size of the output feature maps from the convolutional layers compared to the original architecture [1]. Dropout layers as well as batch normalization layers are added to stabilize the training. The model is trained on a dataset of 30 labeled images which are augmented seven times. Weighted masks for computing the loss are used to compensate for the imbalance of ink pixels vs. background pixels. The output mask computed by the model can be evaluated in terms of, e.g., drop positions, size, and shape.

The image data as well as other existing sensor data is analyzed to define an indicator of the printhead's condition. This indicator is predominantly supposed to help identify the correct labels for the training data used to train the RUL classification model. Possible indicators are e.g. 
the number of ink-drops which deviate from their supposed position greater than a threshold value.

The RUL classification model uses a single long-short-term memory layer followed by a single fully connected layer. The model input consists of geometric properties for each printed drop and relevant sensor data. The dataset, which consists of 940 samples from seven longterm tests, is reduced through a principal component analysis and randomly divided into a training and test set. A random search for optimal hyperparameters is conducted.

\section{Results}

The training process for the image segmentation model clearly converges for both training loss and validation loss. The training process seems to be instable and the model is overfitted since the validation loss is constantly higher than the training loss. Nevertheless, comparing the input images from a test set with the output masks shows that the relevant ink droplets are detected reliably.

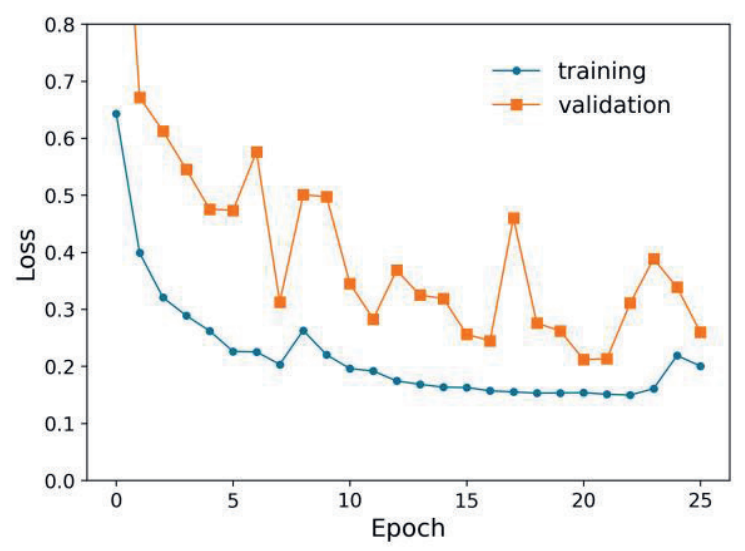

Fig. 1: Training and validation loss over the course of the training process for the image segmentation model.

The features engineered from the resulting information about the ink droplets do not explicitly indicate the time of failure for the printhead. The features often show a time depended increase for a single long-term test but none of the features is applicable to all tests. Therefore, a manual selection of the failure time is conducted to label the long-term-test data as an intermediate solution.

During the training of the RUL prediction model the computed loss converges quickly. Both, training and validation loss are equally low at the minimum after epoch 39. Analyzing the prediction results on the test set of 88 samples show that only two samples are wrongly classified. Furthermore, it shows that the wrongly classified samples are samples from the corresponding neighbor class, e.g. a sample from the class "300 $\min >=$ RUL $>200$ min" is classified to the class "480 $\mathrm{min}>=$ RUL > $300 \mathrm{~min}$ ". The prediction therefore seems to be not random for the misclassified samples.

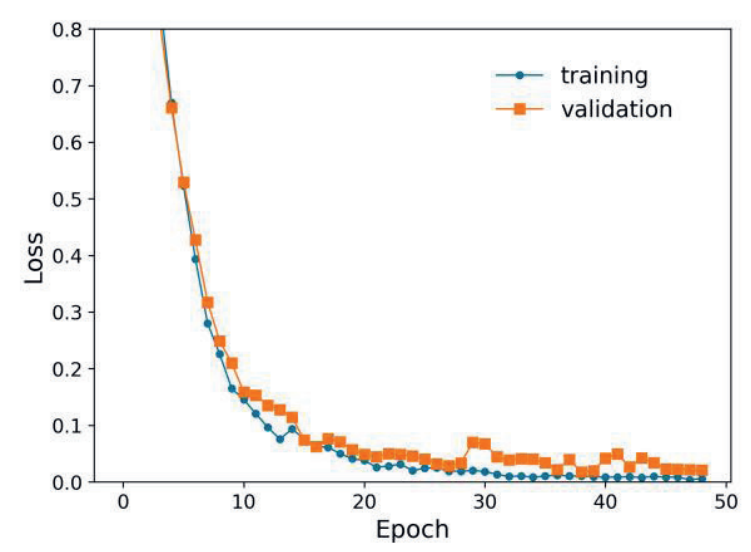

Fig. 2: Training and validation loss for the RULprediction model with LSTM cells.

\section{Discussion}

Our results show that the developed concept is suitable to predict printing failure eight hours in advance with high accuracy. The occurring of overfitting during the training of the U-Net model is likely caused by the model complexity. Since the segmentation task should be relatively easy to achieve by comparing brightness, the number of trainable parameters in the model can cause problems during the training process. Since the validation loss also shows instability, hyperparameter optimization could help overcome both problems. The model is still able to output relevant and useful data.

Further investigation is needed to find an accurate indicator for labeling the RUL-classification data. Nevertheless, manual labeling seems to be accurate enough to train a classification model. More accurate labels as well as more data could possibly lead to a regression model.

\section{References}

[1] O. Ronneberger, P. Fischer, T. Brox, U-Net: Convolutional Networks for Biomedical Image Segmentation, Medical Image Computing and Computer-Assisted Intervention - MICCAI 2015. Lecture Notes in Computer Science, vol 9351 (2015); doi: 10.1007/978-3-319-24574-4_28. 